\title{
Estilos de vida e hipertensión arterial en docentes de la Universidad Nacional del Centro del Perú, Huancayo - 2018
}

\author{
Lifestyles and high blood pressure in teachers of \\ Universidad Nacional del Centro del Perú, Huancayo - 2018
}

'Ortega Salcedo, M.A.; Orellana Salazar, R.M.; Trigos Lara, E.E.; Ramírez Castro, C.

Facultad de Enfermería / Universidad Nacional del Centro del Perú

Email:mortega@uncp.edu.pe

\section{Resumen}

El trabajo universitario, por sus características, podría condicionar la aparición de la hipertensión arterial. El estudio tuvo como propósito determinar la relación entre el estilo de vida y la hipertensión en docentes universitarios de la Universidad Nacional del Centro del Perú (Huancayo) el 2018; con este fin, se realizó un estudio trasversal correlacional considerando una población de 644 docentes de donde se eligió una muestra de 61 docentes que presentaban pre hipertensión o hipertensión.

Entre los resultados se destaca que el $18 \%$ llevan un peligroso estilo de vida y el $31.1 \%$ de los docentes tienen hipertensión arterial alta; asimismo, se encontró que existe una relación inversa entre el estilo de vida en general y la hipertensión arterial (coeficiente de correlación de Spearman - 0.285 y p value 0.026 ); esto indica que la mejora del estilo de vida en general reduce la prevalencia de hipertensión arterial en los docentes.

Palabras Clave: estilo de vida, hipertensión arterial docente universitario

\begin{abstract}
The university work, by its characteristics, could condition the appearance of arterial hypertension. The study had as purpose determining the relationship between the style of life and hypertension in university professors of the Universidad Nacional del Centro del Perú (National University of the Center of the Peru -Huancayo) during 2018, a correlational cross-sectional study was carried out for this purpose, considering a population of 644 teachers from which a sample of 61 teachers who had pre-hypertension or hypertension was chosen.

Among the results, it is highlighted that $18 \%$ have a dangerous lifestyle and $31.1 \%$ of the teachers have high blood pressure, it was also found an inverse relationship between lifestyle in general and hypertension (Spearman correlation coefficient $=-0.285$ and $p$ value $=0.026)$; this indicates that lifestyle improvement in general reduces the prevalence of arterial hypertension in teachers.
\end{abstract}

Keywords: lifestyle, hypertension, university teacher 


\section{Introducción}

La hipertensión, también conocida como presión arterial alta, es el incremento de la presión ejercida por la sangre en los vasos por las cuales fluye, esto hace que el corazón trabaje el doble para circular la sangre a través de los vasos (Burini y Tadashi, 2017).

La hipertensión, que es uno de los principales factores de riesgo cardiovascular, afecta a alrededor del $40 \%$ de los adultos en todo el mundo (Guedes et al., 2014), La hipertensión no controlada ocasiona diversas complicaciones; como, por ejemplo, enfermedad coronaria, derrame cerebral, insuficiencia cardíaca congestiva, insuficiencia renal y enfermedad vascular periférica (Patterson, 2014). La prehipertensión, se define como la presión arterial que se encuentra entre 120 a 139 mm Hg sistólica (el número superior) y de 80 a $89 \mathrm{~mm} \mathrm{Hg}$ diastólica, cualquier cifra que sea de 140/90 mm Hg o más se considera hipertensión (Guedes y Oliveira, 2015).

La hipertensión, se ha convertido en un problema importante en muchos países en desarrollo que experimentan una transición epidemiológica de enfermedades crónicas transmisibles a no transmisibles (Lacruz et al., 2015). También se ha atribuido a la hipertensión, como una de las principales causas de muerte y discapacidad en los países en desarrollo (Fadheel y Hussein, 2017). La aparición de hipertensión y otras enfermedades cardiovasculares, se han convertido en un problema de salud pública en estos países y está fuertemente relacionada con el envejecimiento de las poblaciones, la urbanización y los cambios socioeconómicos que favorecen los hábitos sedentarios, la obesidad, el consumo de alcohol y la ingesta de sal, entre otros (Seham y Samira, 2015). Varias modificaciones de estilo de vida pueden disminuir, prevenir y controlar la hipertensión. Se ha demostrado que el control del peso, la actividad física y una dieta baja en grasas rica en frutas y verduras, con productos lácteos sin grasa y frutos secos incorporados reducen la presión arterial (Shakeel y Irshad, 2017).

El estilo de vida, es un conjunto de patrones de comportamiento identificable y relativamente estable en el individuo o en determinada comunidad, dicho estilo de vida resulta de las interacciones entre las características individuales, la interacción social y las condiciones de vida socioeconómica y ambiental en que viven las personas (Ndanuko et al., 2016). Los estilos de vida, provocan patrones de comportamiento específico que llevan a desarrollar determinadas enfermedades (Sarah, 2017).

Los docentes universitarios llevan largas jornadas de trabajo; además del dictado de clases, cumplen labores de gestión, asesoría, dirección de tesis, tutorías, investigación, publicaciones científicas y académicas, asistencia a congresos, comisiones, preparación de clase y material didáctico, evaluación de exámenes y trabajos, actualización y capacitación, entre otros, sumando a todo lo ante- rior, tienen las exigencias de su vida personal y muchos laboran en otras instituciones para poder cubrir sus demandas familiares (Mandai et al., 2015). Trayendo como consecuencia que la jornada se incremente, sacrificando el tiempo que pudiera destinarlo al descanso, al desarrollo personal o al esparcimiento y la diversión. Todas estas actividades se configuran en un escenario generador de un cúmulo de estresores que afectan a la salud, si no se cuenta con las estrategias apropiadas para afrontarlos cotidianamente (Morales y Teajan, 2015).

Las características del trabajo del docente universitario acarrean la adopción de un estilo de vida poco saludable; casi siempre, se debe de alimentar fuera de casa y consumir alimentos con exceso de grasa y sal, no realiza actividad física de forma regular por las múltiples tareas que debe de cumplir, siempre sobrelleva un entorno laboral de conflicto y tensión, en las actividades sociales se acostumbra consumir bebidas alcohólicas, debe permanecer sentado en una oficina gran parte del tiempo cumpliendo funciones de asesoría y gestión, debe de recortar el tiempo de sueño y descanso para mantenerse actualizado $\mathrm{y}$ atender a todas sus responsabilidades.

El estilo que vida que llevan los docentes universitarios, dado el tipo de trabajo que realizan, condiciona el advenimiento de la hipertensión arterial; sin embargo, muchos docentes podrían ser conscientes del riesgoso estilo de vida que llevan y utilizar múltiples estrategias para controlarla o modificarla, de este modo, los estilos de vida, afectarían poco a la prevalencia de hipertensión arterial en los docentes universitarios.

Además, aquellas personas que padecen hipertensión arterial, llevan un tratamiento farmacológico con el que logran controlar la enfermedad; pero los docentes, ponen poca atención a la modificación de los estilos de vida y siguen con su rutina.

En nuestro medio, no se han encontrado estudios sobre los estilos de vida e hipertensión arterial focalizada en docentes universitarios, considerando que este es un grupo con mayor riesgo para la hipertensión arterial por el peculiar estilo de vida que adquieren.

Por lo mencionado anteriormente, el estudio tuvo como propósito determinar si el estilo de vida del docente universitario, dada la naturaleza de su trabajo, se asocia a la prevalencia de la hipertensión arterial.

Con base en los resultados de este estudio, se propone la creación de un programa universitario para modificar el estilo de vida en los docentes, ofreciendo opciones saludables de alimentación, programas de actividad física, promoción de actividades de recreación y esparcimiento libre de tabaco y bebidas alcohólicas, y desarrollo de competencias para el manejo de situaciones de estrés y tensión en el medio laboral universitario; todo esto, con la finalidad de mejorar el estilo de vida para reducir y/o controlar la 
prevalencia de hipertensión arterial. Asimismo, el estudio advierte que el medio universitario sería generador de un estilo de vida poco saludable para el docente; que, a su vez, está asociado a la prevalencia de hipertensión arterial.

\section{Materiales y métodos}

Se realizó un estudio de tipo observacional, correlacional y transversal, en docentes nombrados en las diversas categorías de la Universidad Nacional del Centro del Perú. Se hizo la medición directa de la presión arterial a los docentes y se les administró un cuestionario para determinar el estilo de vida que llevan. Se trabajó con un solo grupo de docentes procedentes de las 22 facultades de la universidad.

La población de referencia fueron 644 docentes nombrados al año 2018, de donde fueron seleccionados los docentes que aceptaron participar voluntariamente en el estudio, los que laboran dentro del campus universitario, sede principal, y aquellos docentes que presentaban prehipertensión o hipertensión. Se excluyeron a aquellos docentes que tenían alguna patología subyacente diagnosticada. Al final de la selección quedó una muestra de 61 docentes hipertensos participantes del estudio.

Se solicitó, al decanato de cada una de las facultades, la respectiva autorización para realizar el estudio, se ejecutaron reuniones de coordinación e información con las autoridades y los docentes participantes de cada una de las facultades, luego se concertaron las fechas y horas más oportunas para realizar las diversas valoraciones, se les entregó la ficha de consentimiento informado que fue firmado como señal de consentir su participación voluntaria, se valoró la presión arterial y luego rellenaron el cuestionario de estilo de vida; finalmente, se verificó la conformidad en el llenado de los instrumentos.

Para valorar la presión arterial, el esfigmomanómetro aneroide integrado DS44 fue debidamente calibrado, la longitud de la funda del mango fue suficiente para envolver el brazo y cerrarse con facilidad, se retiraron las prendas gruesas y se evitó enrollarlos para que no compriman el brazo. El docente evitó realizar actividad física moderada o intensa en los 30 minutos previos a la medición y estuvo en reposo por lo menos de 5 minutos antes de la toma de la presión; el brazo izquierdo, estuvo descubierto apoyado a la altura del corazón; piernas sin cruzar y pies apoyados cómodamente sobre el suelo. Se evitó hacer la medición en casos de malestar, con vejiga llena, necesidad de defecar, etc. Asimismo, se redujo la ansiedad propiciando un ambiente tranquilo y se minimizó la actividad mental al no hablar y no preguntar. Se tuvo la certeza que se haya evitado el consumo de cafeína, tabaco o fármacos con efecto sobre la presión arterial. La toma de la presión arterial se realizó por tres veces consecutivas con un intervalo de un día entre toma y toma en el trascurso de una semana.
Para valorar el estilo de vida, se utilizó el cuestionario «FANTÁSTICO», que es un instrumento genérico diseñado en el Departamento de Medicina Familiar de la Universidad McMaster de Canadá, que permite identificar y medir el estilo de vida de una población adulta. Este cuestionario contiene 25 ítems cerrados que exploran nueve categorías o dominios físicos, psicológicos y sociales relacionados al estilo de vida, ha sido validada en trabajadores y pacientes de consulta clínica general. Este cuestionario presenta tres opciones de respuesta con valor numérico de 0 a 2 para cada categoría y se miden por medio de una escala tipo Likert, con una calificación de 0 a 100 puntos. Tomando como punto de corte la media de las calificaciones propuestas por los autores del instrumento, cinco niveles de calificación estratifican el estilo de vida: $(<39$ puntos $=$ existe peligro, 40 a 59 puntos $=$ malo, 60 a 69 puntos $=$ regular, 70 a 84 puntos $=$ bueno, 85 a 100 puntos $=$ excelente $)$. Cuanto menor sea la puntuación, mayor es la necesidad de cambio.

Para determinar el estilo de vida, se contabilizaron los puntajes obtenidos y se construyeron tablas de frecuencia de forma global y, para cada una de las dimensiones, se calcularon estadísticos descriptivos.

Para comprobar la asociación entre el estilo de vida y la hipertensión arterial, se calculó el coeficiente de correlación "r de Spearman" de forma general y para cada una de las dimensiones del estilo de vida; para las correlaciones, se utilizó el valor de la PA sistólica. Se utilizó el "p value" como criterio de significancia para las pruebas estadísticas aplicadas ( $\alpha \leq 0.05)$; de manera anticipada, se evaluó la normalidad y la homocedasticidad de los datos. Para el procesamiento de información, se construyó una base de datos con el programa Microsoft Office Excel 2016, que luego fue exportada al programa estadístico SPSS v.24 para Windows, con el que se realizaron los diversos análisis estadísticos.

En la ejecución de la investigación, no se expuso a los docentes a situaciones riesgosas, los cuestionarios rellenados fueron innominados, garantizando la confidencialidad, se aplicó el consentimiento informado como garantía que los docentes han expresado voluntariamente su intención de participar en el estudio, respetando la capacidad de autodeterminación de la persona; quedó claro. que no se les solicitó que participen, sino que les invitó a hacerlo, permitiéndole reflexionar, respondiendo a sus dudas y preguntas, no existió la persuasión difícil de evitar por alguna relación de autoridad.

Con el estudio se alcanzó establecer la asociación significativa entre los estilos de vida y la hipertensión arterial en los docentes; sin embargo, por ser un estudio transversal, no se pudo comprobar la asociación causal bajo el criterio de temporalidad, tampoco se pudieron determinar los otros factores causales de la hipertensión arterial preexistentes en los docentes universitarios. 


\section{Resultados}

En el estudio fueron incluidos 61 docente universitarios; en ellos, se encontró que el $18 \%$ llevan un peligroso estilo de vida y el 57,4 \% tienen un estilo de vida malo. Asimismo, se halló que el $31.1 \%$ de los docentes tienen hipertensión arterial alta y el 59.0\% hipertensión arterial leve [entre los docentes que tenían pre hipertensión o hipertensión] (Figura 1 y 2).

Figura 1

Estilo de vida en docentes de la

Universidad Nacional del Centro del Perú - 2018

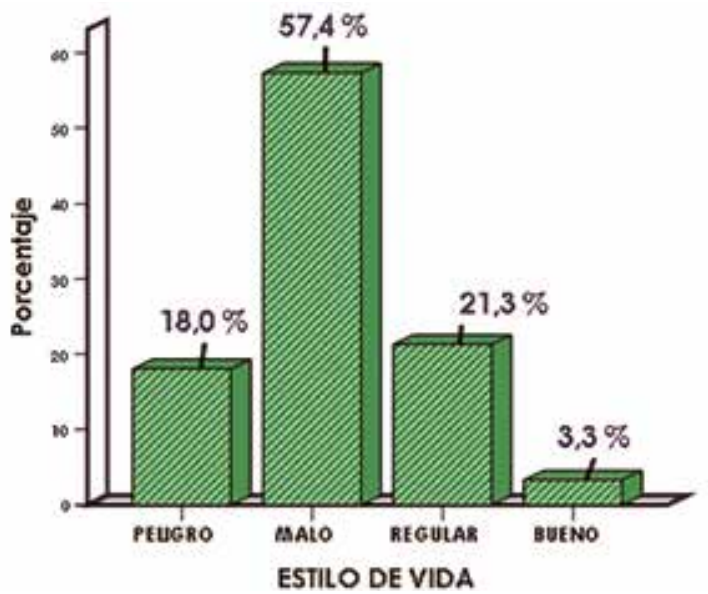

Figura 2

Hipertensión arterial en docentes de la Universidad Nacional del Centro del Perú - 2018

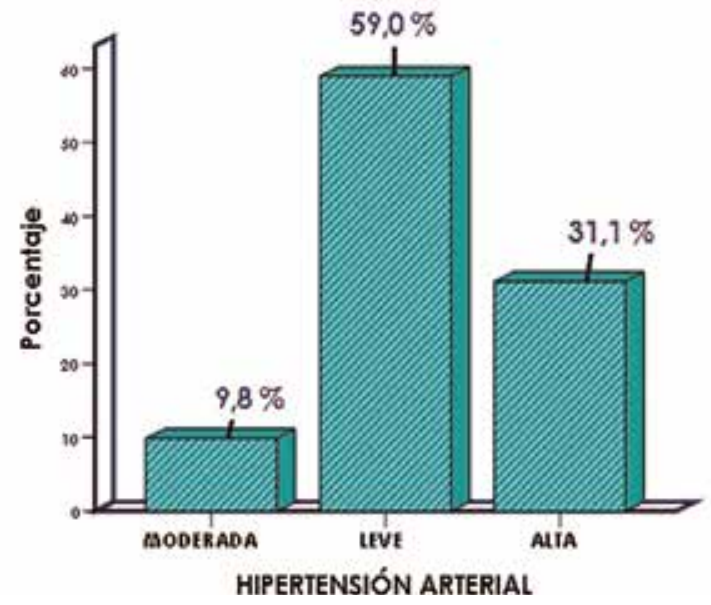

Asimismo, se remarca que el $21.3 \%$ de los docentes, tienen un estilo de vida nutricional peligroso y el 54.1 $\%$, es malo; el $18 \%$, lleva un estilo de vida en ejercicios peligroso y el $52.5 \%$, es malo; el $18 \%$, lleva un estilo de vida de sueño y reposo peligroso y en el $49.2 \%$ es malo; el $8.2 \%$ lleva un estilo de vida en adicción peligroso y en el $54.1 \%$ es malo (Figura 3 ).

Figura 3

Estilo de vida en docentes de la Universidad Nacional del Centro del Perú - 2018

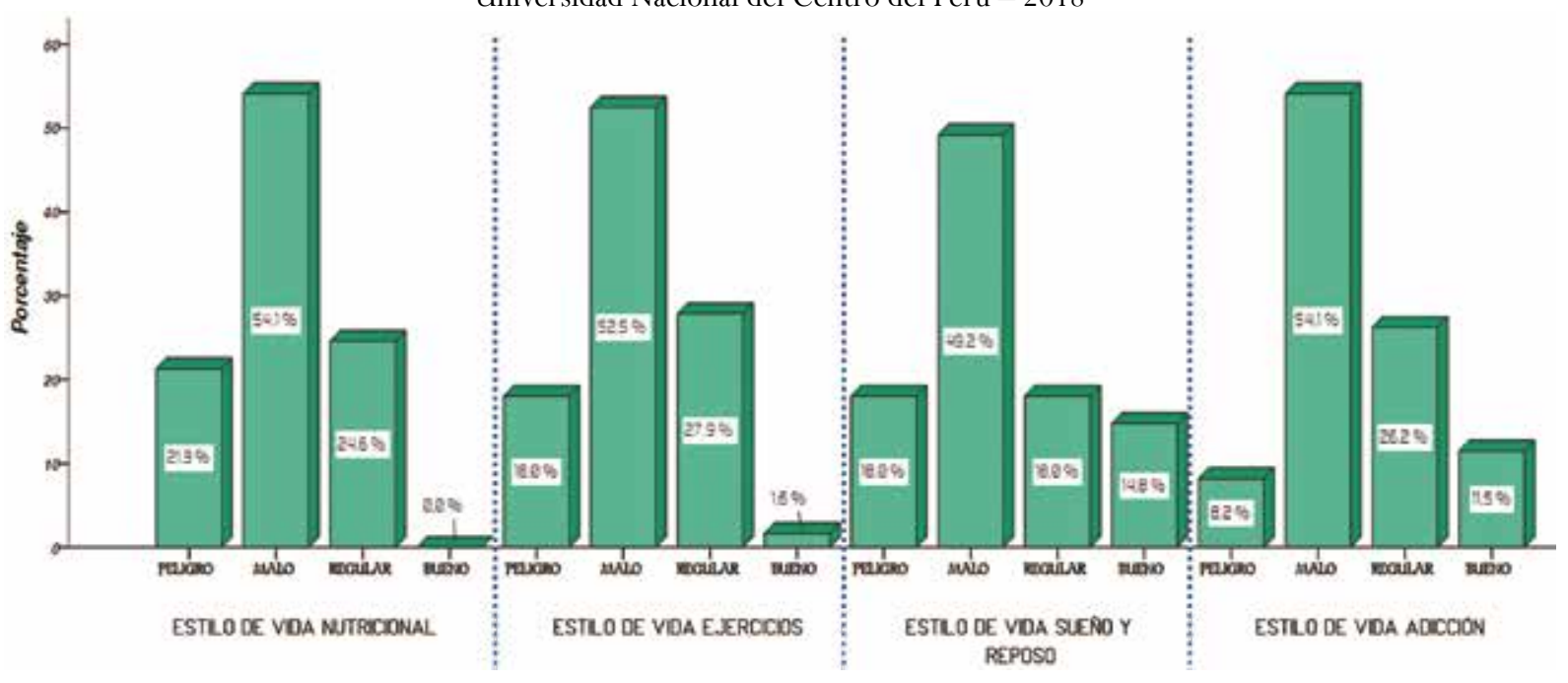

Se encontró que la relación entre el estilo de vida en general y la hipertensión arterial, es inversa y significativa con un coeficiente de correlación de Spearman de -0.285 y un p value de 0.026. Esto indica que, cuando mejora el estilo de vida en general, se reduce la prevalencia de hipertensión arterial (Tabla 01).

Así también, se destaca que la relación entre el estilo de vida nutricional y la hipertensión arterial es inversa y significativa con un coeficiente de correlación de Spearman de - 0.276 y un p value de 0.032 . Esto indica que, cuando mejora el estilo de vida nutricional, se reduce la prevalencia de hipertensión arterial. De forma similar, para el estilo de vida en ejercicios, la $r$ de Spearman es de -0.282 y un $p$ value de $0.028 \mathrm{y}$, para el estilo de vida en sueño y reposo, la $\mathrm{r}$ de Spearman es de - 0.257, con un $\mathrm{p}$ value de 0.046 (Tabla 1 ). 
Tabla 1

Correlación entre estilo de vida e hipertensión arterial en

docentes de la Universidad Nacional del Centro del Perú - 2018

Correlaciones

\begin{tabular}{ccccccc}
\hline & & $\begin{array}{c}\text { Estilo de vida } \\
\text { en general }\end{array}$ & $\begin{array}{c}\text { Estilo de vida } \\
\text { nutricional }\end{array}$ & $\begin{array}{c}\text { Estilo de vida } \\
\text { en ejercicios }\end{array}$ & $\begin{array}{c}\text { Estilo de vida } \\
\text { de sueño y } \\
\text { reposo }\end{array}$ & $\begin{array}{c}\text { Estilo de vida } \\
\text { en adicción }\end{array}$ \\
& Rho de Spearman & -0.285 & -0.276 & -0.282 & -0.257 & -0.225 \\
Hipertensión arterial & Sig. (bilateral) & 0.026 & 0.032 & 0.028 & 0.046 & 0.082 \\
& $\mathrm{~N}$ & 61 & 61 & 61 & 61 & 61 \\
\hline
\end{tabular}

**. La correlación es significativa en el nivel 0,01 (bilateral).

\section{Discusión}

En el estudio se encontró que; cuando mejora el estilo de vida en general, se reduce la prevalencia de hipertensión arterial $(\mathrm{r}$ de Spearman $=-0.285 ; \mathrm{p}$ value $=0.026)$.

Esto se debe a que el inadecuado estilo de vida que llevan los docentes, caracterizado por la inactividad física, insuficiente descanso y sueño y los malos hábitos alimenticios, son los principales factores de riesgo para la hipertensión; si embargo, los mecanismos exactos de la relación entre un estilo de vida y la hipertensión no están completamente claros (Mandai et al., 2015). Sobre la base de la fuerte relación individual de la hipertensión, con tener un índice de masa corporal alto está involucrada la disfunción endotelial, la rigidez arterial, la disfunción de los barorreceptores y el aumento de la actividad simpática, como mecanismos que potencialmente conducen a una presión arterial, pero la actividad física no se asocia de forma independiente con la hipertensión en el presente estudio (Maseli et al., 2017) (Khalesi et al., 2018).

De forma similar Patnaik (2017), halla una asociación inversamente proporcional entre los estilos de vida y la hipertensión arterial; afirma que, con solo una modificación adecuada de la dieta, las modificaciones dietéticas y evitar la situación estresante, pueden obviar totalmente la necesidad de un tratamiento farmacológico. La intervención en forma de educación sobre el estilo de vida de los hipertensos es útil para aumentar la conciencia sobre los factores de riesgo modificables, esto les permitirá adoptar y practicar un estilo de vida saludable, lo que ayudará a controlar la presión arterial y evitar complicaciones relacionadas con la hipertensión (Ortega et al., 2016) (Okwuonu, Emmanuel y Ojimadu, 2014).

Asimismo, Njambi (2014), encuentra una asociación significativa entre las modificaciones del estilo de vida y la disminución de la prevalencia de la hipertensión arterial, afirma que la modificación del estilo de vida, también conocida como terapia no farmacológica, es la piedra angular para ayudar a los pacientes hipertensos a lograr comportamientos de estilo de vida que sean saludables; recomienda, un estilo de vida más saludable para minimizar el riesgo de hipertensión con una combinación de fármacos antihipertensivos. Los factores de estilo de vida que se sugieren para reducir la hipertensión, incluyen pérdida de peso, ejercicio físico regular, consumo moderado de alcohol, cambios en la dieta y consumo reducido de sodio. Los enfoques dietéticos, para detener la hipertensión, recomiendan muchas frutas y verduras, productos lácteos bajos en grasa, además del mantenimiento del peso corporal normal, es importante realizar un entrenamiento de 30 minutos al menos 5 días a la semana. También evidencia, que el exceso de sal se asocia con la elevación de la presión arterial alta, por lo que se propone la restricción de sal (Siyum et al., 2017) (Marfo, Owusu-Daaku, Addo y Saana, 2014).

Así también, Bruno y Amaradio (2018), encuentran una asociación significativa entre la modificación de los estilos de vida y el control de la hipertensión arterial; entre los componentes del estilo de vida, remarcan la importancia de la actividad física, indican que el ejercicio físico juega varios efectos beneficiosos hemodinámicos y metabólicos. Por otro lado, Verma, Manushi y Ratan (2015), manifiestan que reduce las respuestas simpáticas y afecta el eje hipotalámico-hipofisario-suprarrenal con un menor aumento del cortisol, una menor reactividad cardiovascular y una recuperación cardiovascular más rápida en respuesta al estrés psicofísico.

Además, la actividad física, determina una adaptación sistémica de la pared arterial que podría llevar a una disminución de la resistencia periférica. El entrenamiento con ejercicios, lleva a un mayor número de capilares para la fibra muscular al aumentar una serie de factores pro angiogénicos, la actividad física, también puede mejorar la función vascular al reducir la rigidez arterial y mejorar el equilibrio entre los sistemas vasoconstrictor y vasodilatador (Hareri, Gedefaw y Simeng, 2014) (Elbur, 2015). 
De la misma manera, las personas con prehipertensión y la hipertensión, que modificaron su estilo de vida, mejoraron el control de la presión arterial y mejoraron su calidad de vida (Fathia y Nahed, 2015). Las variaciones en el estilo de vida, que abordó el control del peso, la limitación del consumo de alcohol, el aumento de la actividad física, el aumento del consumo de frutas y verduras, la reducción de la ingesta total de grasas saturadas y el abandono del hábito de fumar; logaron reducir de forma significativa la presión arterial y llevar un normal ritmo de vida (Blumenthal y Sherwood, 2015).

La implementación de las pautas de estilo de vida, mejoran la autoeficacia que incide en un mejor autocuidado de sí mismo y en el control de la hipertensión arterial (Hinderliter, Sherwood y Craighead, 2014). La modificación del estilo de vida que implicaba: reducir el consumo de sal en la dieta e incrementar el consumo de vitamina $C$, calcio y fibra, además de reducir las grasas, carne roja, dulces y las bebidas azucaradas, reemplazándolos con granos integrales, carne de aves, productos lácteos bajos en grasas, pescado y frutos secos; llevaba al control de hipertensión arterial, incluso se podría prescindir de los medicamentos antihipertensivos (Blumenthal y Sherwood, 2018) (Durai y Muthuthandavan, 2015).

Mejorar el estilo de vida con la abundancia de alimentos vegetales: frutas, verduras, cereales, papas, legumbres, frutas secas, aceite de oliva; queso, yogurt, pescado y pollo, escasas carnes rojas y evitar la adición de sal, eludir los alimentos con exceso de sal (elaborados); alivia la hipertensión arterial (Samadian y Dalili, 2016). Un estilo de vida con frecuente actividad física y evitando el estrés, la ansiedad y la ira, se asocian al adecuado nivel de presión arterial (Ghanbari y Mohammadpoorasl, 2018).

Evaluar el papel del estilo de vida en hipertensión, es realmente difícil, porque existen muchos factores de confusión, como la edad, la magnitud y la duración del ejercicio, los músculos involucrados, el tipo de alimento, el estrés la ansiedad, etc. y las comorbilidades que pueden llevar a una falta de coincidencia de los resultados.

A pesar que los estudios informan asociaciones significativas entre el estilo de vida y la hipertensión arterial, los resultados son difíciles de comparar porque se obtuvieron con diferentes metodologías, bajo diferentes condiciones y en poblaciones distintas.

Basados en los resultados de este estudio, aconsejamos la creación de un programa universitario para modificar el estilo de vida en los docentes, ofreciendo opciones saludables de alimentación, programas de actividad física, promoción de actividades de recreación y esparcimiento libre de tabaco y bebidas alcohólicas y desarrollo de competencias para el manejo de situaciones de estrés y tensión en el medio laboral universitario; todo esto con la finalidad de mejorar el estilo de vida para reducir y/o controlar la prevalencia de hipertensión arterial.

\section{Conclusiones}

- La mayor parte de los docentes tienen un inadecuado estilo de vida y a la vez presentan moderada hipertensión arterial, se ha verificado la asociación del estilo de vida y la hipertensión arterial (Rho de Spearman $=-0.285 ; \mathrm{p}$ value $=0.026)$; se necesita plantear programas de modificación de los estilos de vida y comprobar su efectividad.

- Es conveniente implementar programas que modifiquen el estilo de vida, ofreciendo opciones de alimentación, actividad física y actividades de esparcimiento saludables, el que, además, debe de asegurar la participación masiva de los docentes.

\section{Referencias bibliográficas}

Blumenthal J, Sherwood A,. (2015). Lifestyle modification for resistant hypertension: The TRIUMPH randomized clinical trial. Am Heart J, 170(5), 986 - 994.

Blumenthal J, Sherwood A, . (2018). Missing ingredients for a lifestyle recipe to treat hypertension. J Thorac Dis, 10(2), 653 - 656.

Bruno M, Amaradio M,. (2018). Lifestyle and hypertension: An Evidence-Based review. journal of hypertension and management, 4(1), 1 - 10.

Burini R, Tadashi H,. (2017). The lifestyle modication effectiveness in reducing hypertension in a brazilian community: From the epigenetic basis of Ancestral Survival to the contemporary lifestyle and public health initiatives. Heighpubs Journal of Clinical Hypertension, 10(2), 10 - 31.

Durai V, Muthuthandavan A,. (2015). Knowledge and practice on lifestyle modifications among males with hypertension. Indian J Community Health, 27(1), $143-149$.

Elbur A. (2015). Level of adherence to lifestyle changes and medications among male hypertensive patients in two hospitals in Taif; Kingdom of Saudi Arabia. Int J Pharm Pharm Sci, 7(4), 168 - 172.

Fadheel Q, Hussein A,. (2017). Prevalence and life style determinants of hypertension among women in Iraq. Open Access Journal of Pharmaceutical Research, 11(4), 1 - 8 .

Fathia A, Nahed A,. (2015). Effect of evidence based lifestyle guidelines on self efficacy of patients with hypertension. Int.J.Curr.Microbiol.App.Sci, 4(3), $244-263$.

Ghanbari J, Mohammadpoorasl A,. (2018). Subgroups of lifestyle patterns among hypertension patients: a 
latent-class analysis. BMC Medical Research Methodology, 18(127), 1 - 8.

Guedes L, et al,. (2014). Nursing diagnosis sedentary lifestyle in individuals with hypertension: an analysis of accuracy. Rev Esc Enferm USP, 48(5), $803-808$.

Guedes L, Oliveira V,. (2015). Sedentary lifestyle in individuals with hypertension. Rev Bras Enferm, 68(6), $697-704$.

Hareri HA, Gedefaw M, Simeng B, . (2014). Assessment of prevalence and associated factors of adherence to antihypertensive agents among adults on follow up in Adama Referal hospital, east Shoa, Ethiopia-cross sectional study. Int J Curr Microbilol App Sci, 3(1), $760-770$.

Hinderliter A, Sherwood A, Craighead L,. (2014). The Long-Term effects of lifestyle change on blood pressure: One-year follow-up of the ENCORE study. American Journal of Hypertension, 27(5), 734 - 741 .

Khalesi S, et al, . (2018). Lifestyle and self-management determinants of hypertension control in a sample of Australian adults. Expert Review Of Cardiovascular Therapy, 25(3), 1 - 8.

Lacruz M, Kluttig A, Hartwig S, Löer M, Tiller D, Greiser K, et al,. (2015). Prevalence and incidence of hypertension in the general adult population: Results of the CARLA-Cohort Study. Medicine (Baltimore), 94(22), 952 - 958.

Mandai N, Akazawa K, Hara N, Ide Y, Ide K, Dazai U, et al,. (2015). Body weight reduction results in favorable changes in blood pressure, serum lipids, and blood sugar in middle-aged japanese persons. Glob J Health Sci, 7(5), 159 - 170.

Marfo A, Owusu-Daaku F, Addo M, Saana I,. (2014). Ghanaian hypertensive patients understanding of their medicines and lifestyle modification for managing hypertension. Int. J Pharm Pharm Sci, 6(4), $165-170$.

Maseli A, Aeschbacher S, et al,. (2017). Healthy lifestyle and blood pressure variability in young adults. American Journal of Hypertension, 20(7), 690 - 699.

Morales N, Teajan M, . (2015). Promocion de salud en docentes universitarios. Proceso psicológicos y sociales, $17(2), 52-63$.

Ndanuko R, Tapsell L, Charlton K, Neale E, Batterham M,. (2016). Dietary pat terns and blood pressure in adults: A systematic review and meta-analysis of randomized controlled trials. Adv Nutr, 7(1), 76 - 89.
Njambi O, T. A. (2014). Lifestyle modification in prevention of hypertension: Patient empowerment. [Tesis], Universidad de Ciencias Aplicadas de Seinäjoki, Degree programme in Nursing.

Okwuonu C, Emmanuel C, Ojimadu N,. (2014). Perception and practice of lifestyle modification in the management of hypertension among hypertensive in south-east Nigeria. Int J Med Biomed Res, 3(2), $121-131$.

Ortega R, et al,. (2016). Pautas nutricionales en prevención y control de la hipertensión arterial. Nutr Hosp, 33(4), 53 - 58.

Patnaik L, K. K. (2017). Lifestyle pattern and hypertension related knowledge, attitude and practices among diagnosed patients of hypertension attending a tertiary care Hospital. J Cardiovasc Disease Res, 8(4), $108-111$.

Patterson A. (2014). Evaluating the effects of an educational lifestyle modification intervention on blood pressure in adults with prehypertension. [tesis], University of North Florida, School of medicine.

Samadian F, Dalili N,. (2016). Lifestyle modifications to prevent and control hypertension. Iranian Journal of Kidney Diseases, 10(5), 237 - 263.

Sarah M, S. J. (2017). Lifestyle determinants of hypertension among female school teachers. Int. J. of Life Sciences, 5(4), 696 - 702.

Seham A, Samira E,. (2015). Knowledge and perceptions related to hypertension, lifestyle behavior modifications and challenges that facing hypertensive patients. Journal of Nursing and Health Science, 4(6), 15 - 26.

Shakeel S, Irshad N,. (2017). Lifestyle patterns and the prevalence of hypertension among the teachers of Kashmir University (Age 35 to 60 Yrs.). International Journal of Home Science, 3(1), $150-154$.

Siyum E, et al,. (2017). Lifestyle modification practice and associated factors among diagnosed hypertensive patients in selected hospitals, South Ethiopia. Clinical Hypertension, 23(26), 2 - 9.

Verma P, Manushi S, Ratan K,. (2015). Assessment of extent of lifestyle modification among diagnosed patients of hypertension attending tertiary care hospital. Int $J$ Med Health Sci, 4(2), $196-201$. 